\title{
Mythos
}

Rivista di Storia delle Religioni

$13 \mid 2019$

Varia

\section{La rivolta come fenomeno religioso. Considerazioni attorno alla lettura jesiana del sabbatianesimo}

The revolt as a religious phenomenon. Considerations about Jesi's reading of Sabbatianism

\section{Marco Tabacchini}

\section{OpenEdition}

\section{Journals}

\section{Edizione digitale}

URL: http://journals.openedition.org/mythos/1158

ISSN: 2037-7746

\section{Editore}

Salvatore Sciascia Editore

Notizia bibliografica digitale

Marco Tabacchini, « La rivolta come fenomeno religioso. Considerazioni attorno alla lettura jesiana del sabbatianesimo », Mythos [Online], 13 | 2019, online dal 22 octobre 2019, consultato il 25 décembre 2019. URL : http://journals.openedition.org/mythos/1158

Questo documento è stato generato automaticamente il 25 dicembre 2019

Mythos 


\title{
La rivolta come fenomeno religioso. Considerazioni attorno alla lettura jesiana del sabbatianesimo
}

\author{
The revolt as a religious phenomenon. Considerations about Jesi's reading of
}

Sabbatianism

Marco Tabacchini

In effetti il permanere dell'esistente è la catastrofe. W. Benjamin ${ }^{1}$

1. Pubblicato nel 1971 e ripreso l'anno seguente nel volume Mitologie intorno all'illuminismo, il saggio intitolato Il miracolo secondo ragione $e^{2}$ sembra porsi, per più di una ragione, in continuazione con la fenomenologia religiosa che Furio Jesi aveva precedentemente avanzato nel manoscritto di Spartakus ${ }^{3}$. Secondo un gesto squisitamente jesiano, per il quale i materiali offerti dalla tradizione letteraria e storica costituiscono altrettanti terreni di ricognizione entro i quali vagliare la tenuta delle proprie costruzioni gnoseologiche, l'indagine qui condotta attorno all'eresia sabbatiana si presta ancora una volta a proseguire l'interrogazione circa il peso con cui l'orizzonte mitologico grava sui gesti di rivolta. E così, pur variando il terreno d'indagine, scelto stavolta nella sua lontananza temporale e senza ricorrere alla mediazione degli autori di lunga frequentazione (quali furono Benjamin, Brecht o Eliade in Spartakus), non solo vi vengono ribaditi quei nessi tra etica ed esilio dalla storia, o tra rivolta e nichilismo, precedentemente affrontati seguendo i passi dell'insurrezione spartachista, ma tali vincoli tornano a configurarsi come altrettanti nessi tra azione politica ed esperienza religiosa. Non è un caso che, proprio in questo saggio, Jesi si sia arrischiato a formulare un concetto di "religione" all'altezza dell'uso tecnico con cui questo è impiegato, riassunto nella definizione seguente: "Ogni religione è un complesso di organismi viventi adunati intorno a manifestazioni di forze e collegati fra loro in modo che le esperienze anche di una piccolissima minoranza di essi si ripercuotano profondamente sulla totalità» ${ }^{4}$. In tal senso, precisa Jesi, simili complessi «sono organizzati attorno a manifestazioni di forze e appaiono caratterizzati dalle diverse relazioni con tali 
epifanie [...] relazioni suscettibili entro certi limiti, di descrizione storica e quindi di interpretazioni che vi riconoscano "condizionamenti storici", ma anche relazioni (o qualità di esperienze religiose) che propongono un senso della storia» ${ }^{5}$. Se nelle riflessioni condotte in Spartakus era riconosciuto un posto di prim'ordine a simili condizionamenti storici, il peso dei quali avrebbe comportato la radicale inattualità della rivolta, ora l'accento è maggiormente posto sulle esperienze religiose e sul senso della storia che a esse si accompagna e che da esse procede. Centrale, in entrambi i casi, è l'attenzione rivolta al «ricorso al mito inteso come pratica vissuta di forme mitologiche $»^{6}$ da parte di soggetti interessati a saldare prassi religiosa e prassi politica nella fruibilità immediata di un eterno presente: il dopodomani realizzato, la redenzione compiuta e l'utopia mitologica; in altri termini, il tempo del mito fattosi tempo della storia. In tal senso, se il saggio sembra proporsi quale riflessione sulle tensioni che intercorrono fra l'ebraismo rabbinico ortodosso e la sua versione eretica, l'analisi del sabbatianesimo, col suo antinomismo più volte associato a posizioni marraniche, serve piuttosto a chiarire il rapporto che l'agire umano instaura tra il tempo della storia in cui si compie e il tempo del mito a cui non cessa di tendere. Indagando le reciproche interrelazioni tra forme di esperienza religiosa e la contingenza storica in cui esse non possono che dispiegarsi, Jesi suggerisce di cogliere la compiuta sovrapposizione tra mistica e politica entro un ambito nel quale «non si trattava di operare politicamente alla luce delle esperienze interiori, ma di operare politicamente nelle esperienze interiori, nel cuore stesso dell'esperienza mistica. Il messianismo e l'antinomismo dei sabbatiani non hanno conseguenze politiche, ma sono azioni politiche nell'istante in cui sono esperienze mistiche» ${ }^{7}$.

2 Con il sabbatianesimo è la stessa vicenda del rapporto tra religione e mito ad assurgere a compiuta leggibilità, là dove il caso estremo sembra saldare inscindibilmente tra loro anelito di riscatto e accettazione del nichilismo. Seguendo un'intuizione già cara a Gershom Scholem, secondo la quale esisterebbe un legame privilegiato tra religione e nichilismo, Jesi ripercorre i concetti fondamentali della mistica ebraica alle soglie dell'Illuminismo a partire dalla torsione impressa loro dal sabbatianesimo, allo scopo di mostrare la necessità di considerare il nichilismo non solo come fenomeno politico e religioso a un medesimo tempo, ma come modalità privilegiata d'accesso per indagare $\mathrm{i}$ comuni punti di giunzione tra le due sfere. Non sorprende che siano proprio gli studi scholemiani il diretto riferimento per la scrittura di Jesi, a riconferma di una felice consonanza tra le posizioni dei due studiosi attorno alla questione del mito: il tentativo, perseguito da Scholem lungo tutto il corso della sua ricerca, di riconciliare l'apparente opposizione tra mito e ragione, tra mistica e istituzione in seno alla tradizione ebraica, nonché l'aporetica condizione del mestiere di storico, costretto a confrontarsi con una verità occultata dalla stessa tradizione che la custodisce, trovano riscontro nelle stesse modalità jesiane con cui i materiali mitologici sono affrontati e circoscritti. Lo stesso obiettivo del volume Mitologie intorno all'illuminismo, ossia quello di restituire all'illuminismo quella componente mitologica che lo attraversa sotterraneamente tanto da instillarvi i germi del «contrasto fra la legge per eccellenza e il tempo storico»" ha il suo diretto antecedente nella concezione scholemiana di una mitologia ebraica che, lungi dal permettere una via di fuga dalla storia, ne costituisce al contrario una torsione essenziale e inaggirabile ${ }^{10}$. Ed è ancora da Scholem, particolarmente attento alle implicazioni teologico-politiche di ogni tentavo mitologico-messianico di rottura con l'ordine esistente ${ }^{11}$, che Jesi mutua il proposito di descrivere l'eresia sabbatiana sotto i tratti di un inaudito gesto di rivolta contro i valori 
legati a una tradizione sempre più incline a ricondurre l'ebraismo al principio dell'osservanza della legge. Episodio unico per tragicità e conseguenze nella storia religiosa ebraica, per entrambi gli studiosi «il sabbatianesimo rappresenta la prima seria rivolta in seno all'ebraismo, a partire dal medioevo» ${ }^{12}$ : una rivolta che avvampa entro le mura del getto e che si alimenta tanto delle sue speranze ed esperienze quanto dei suoi stessi edifici concettuali, ma con il rischio di farli crollare fin dalle fondamenta.

3 La vicenda sembra inizialmente racchiudersi entro le gesta di Shabbetày Tzevì, nato a Smirne nel 1626 ed esplicitamente proclamatosi, quattro decenni dopo, messia e guida di un'«eresia mistica» ${ }^{13}$ dal carattere rivoluzionario e antinomico. Il suo successo fu tale che il risveglio messianico così provocato diede origine a un vero e proprio movimento ${ }^{14}$, grazie a una diffusa atmosfera di intensa e fiduciosa impazienza nell'imminenza della redenzione - impazienza contestatrice dell'indefinita attesa che, secondo la tradizione rabbinica, certo preparava la venuta del messia ma senza offrire alcuna seria aspettazione di vederla. D'altra parte, quale movimento proiettato verso la «distruzione di ogni autorità in nome dell'esperienza mistica o della stessa illuminazione $»^{15}$, esso si prestava a ricevere le sollecite attenzioni di ogni forma di potere costituito, religioso o politico. Così come l'ebraismo rabbinico, intransigente verso ogni forma di contestazione, avrebbe mal tollerato «una rivoluzione della coscienza ebraica che minacciava di distruggere gli antichi valori nelle fiamme del messianismo» ${ }^{16}$, anche il sultanato si sarebbe presto dimostrato altrettanto preoccupato dall'instabilità sociale che la tensione messianica contribuiva ad alimentare. $\mathrm{E}$ ciò non solo perché il movimento sorse, fino a un certo punto, in risposta agli eventi storici contemporanei al suo emergere e consolidarsi, come i pogromy che dal 1648 colpirono a più riprese le comunità ebraiche dell'Europa orientale e che contribuirono a donare un significato urgente ad ogni inclinazione apocalittica. Capace di convogliare nella sua figura le più diverse aspirazioni e tradizioni viventi in seno all'ebraismo, il nichilismo mistico inaugurato da Tzevì seppe infatti coniugare catastrofe e redenzione nel tentativo di «realizzare quel superamento dell'attualità storica in funzione di un compimento messianico» ${ }^{17}$.

4 Nemmeno la conversione all'Islam di Tzevì, costretto ad abiurare la propria fede sotto pressione del sultano ottomano, bastò a frenare l'entusiasmo dei suoi seguaci. L'apostasia fu infatti accolta, entro un contesto escatologico di trasvalutazione dei valori, come una scelta provvidenziale che avrebbe permesso al messia di compiere attivamente la sua missione discendendo nell'impurità mondana ${ }^{18}$. Non solo: per alcuni sabbatiani radicali, meno inclini ad attendere passivamente il ritorno trionfante del Messia, il paradosso della conversione avrebbe costituito un doveroso esempio, mentre il marranesimo volontario che ne sarebbe giocoforza seguito sarebbe stato il segno del destino ebraico relativo alle ultime e più impervie tappe dell'esilio, là dove l'intero popolo eletto avrebbe potuto ritorcere il male contro se stesso e debellarlo dall'interno.

5 2. Sarebbe arduo cogliere la specificità del movimento sabbatiano limitandosi a registrarne l'antinomismo, senza che questo sia debitamente associato al preciso supporto mitologico in grado di restituirgli coerente leggibilità. In grado di designarlo, dunque, quale diretta continuazione, e quasi compimento, delle grandi correnti del messianismo ebraico, prima tra tutte la scuola di Safed e la Qabbalah di Luria, da cui l'eresia mutuò non soltanto i principali concetti e le più importanti narrazioni, ma anche la sua straordinaria adattabilità alle urgenze della situazione storica contingente. Scholem, da parte sua, non ha mancato di insistere sul fatto che il cuore dell'impresa 
teologica di Luria consistette anzitutto nell'inscrivere un evento storico di indubitabile portata, come la cacciata degli ebrei dalla Spagna a partire dal 1492, entro un contesto squisitamente mitologico, a tal punto che «l'innovazione decisiva, quella che fece perdurare il segreto del fascino luriano, [fu] la trasposizione dei concetti fondamentali di esilio e redenzione da un piano storico a un piano cosmico e addirittura divino» ${ }^{19}$. Rispetto alla tradizione ebraica ortodossa, da sempre contraddistintasi per la sua tendenza a liquidare il mito al fine di garantire la purezza del concetto di Dio rispetto ad ogni narrazione mitologica, qui Dio si trova inscritto entro la costruzione di un'ontologia radicalmente mitologica, una speculazione in grado di coniugare armonicamente le vicende cosmiche con quelle della storia intramondana, di cui le prime ne costituiscono il parallelo e perfino il modello o il destino. Fu proprio in favore di una simile inscrizione nel tempo del mito che la Qabbalah di Luria finì per consegnare l'evento storico a una narrazione mitologica tutta tesa a fare del Tiqqun, la restaurazione dello splendore divino nel mondo, il punto nodale di un intreccio in grado di congiungere teologia e politica, tanto che il mito dell'esilio quale «mito nazionale popolare rivestì qui sfumature e colori nuovi, dilatandosi sino a diventare dramma cosmico» ${ }^{20}$.

Dottrina centrale della Qabbalah di Safed è quella rubricata sotto il concetto di Tzimtzùm , una delle idee mistiche più efficaci della teologia di Luria, con la quale lo stesso tentò di opporre, all'idea di una creazione divina dal nulla, la concezione dell'universo come originatosi da un processo di contrazione di Dio in se stesso: «il cabbalistico Tzimtzùm non significa la concentrazione di Dio in un luogo, ma il suo ritrarsi fuori da ogni luogo [...]. Dio - per garantire la possibilità del mondo - dovette rendere vacante nel suo essere una zona, dalla quale Egli quindi si ritrasse ${ }^{21}$ esiliandosi da quanto con quel gesto stesso stava creando. La precisazione non è causale, e anzi, seguendo le indicazioni dello stesso Scholem là dove propone di tradurre il termine con "ritiro" anziché con "concentrazione" o "contrazione", esso permette di concepire l'idea dello Tzimtzùm come il più perfetto simbolo dell'esilio stesso, di un esilio (Galuth) in cui Dio stesso si ritira dal mondo nel fondo del suo stesso essere. La tendenza della tradizione ebraica classica a liquidare le proprie componenti mitologiche riconfermando ad ogni occasione «il distacco della legge da ogni esecuzione cosmica»" ${ }^{22}$, trova qui la sua più importante contestazione mistica entro un evento di portata cosmica in grado di colpire Dio stesso; e così, proprio in favore di questa stretta corrispondenza tra la condizione divina e il mondo da essa creato, lo Tzimtzùm potrà essere facilmente presentato come un simbolo in grado di accomunare la condizione di tutte le cose, sia quelle intramondane sia quelle riguardanti le stesse potenze divine. Da qui prenderà nuovamente le mosse, e con rinnovato vigore, quel grande mito dell'esilio in grado di offrire una trasparenza simbolica tanto all'origine del cosmo quanto alle vicende storiche dell'uomo in quanto tale (e non solo del popolo eletto). E così, a partire da quell'atto originario, non soltanto la luce divina, non soltanto le anime, ma ogni essere finisce per mostrarsi esiliato. Persino la Torah stessa avrebbe condiviso una medesima sorte, poiché anche la sua parola sarebbe stata oscurata in tempo di esilio, mentre i suoi segreti si sarebbero trovati irrimediabilmente sigillati fino alla vigilia delle redenzione. Solo allora, grazie all'intervento di un messia in grado di restituirne la corretta leggibilità, essa avrebbe acquisito la sua originaria e definitiva chiarezza rivelando infine i suoi segreti più reconditi e le sue verità non velate dal peccato.

7 Una volta posta al cuore della cosmogonia ebraica la concezione per cui «qualcosa di Dio è bandito dal suo Essere» ${ }^{23}$, ben si comprende come tutti i successivi atti della 
teodrammatica luriana - l'emanazione della luce divina e la conseguente "rottura dei vasi", il fallimento degli Adàm, la dispersione della Shekhinah nel mondo terreno e la decisiva azione umana per risollevare le scintille - si siano svolti nel rispetto di una scena inaugurale a partire dalla quale «nulla rimane al suo posto, nulla resta come sarebbe dovuto essere, e più niente quindi è nella sua debita sede. Tutto è in esilio»" ${ }^{24}$. Il Tiqqun come "riparazione" o "restaurazione" altro non sarebbe che il processo di restituzione dell'esistente a quella condizione a cui originariamente avrebbe mirato la creazione stessa, il ritorno di tutte le cose al posto che avrebbero dovuto occupare nello schema universale; un processo che, se da un lato si presenta come cosmico, dall'altro non può non investire la storia del mondo e il compito, politico e religioso a un tempo, che in esso l'uomo reca con sé.

8 Approfondendo il senso dell'esperienza storica dell'ebreo in esilio secondo i tratti di una vera e propria missione, di un'elezione entro lo stesso evento divino della creazione - così come le scintille divine sono sparse in tutto il mondo, allo stesso modo frammenti di scintille messianiche sono presenti in ogni ebreo - , a questi non spettava altro che accogliere la carica messianica in lui riposta e compierla nei gesti più quotidiani: dal momento in cui la grande epopea dell'esilio e della redenzione si svolge anche nello spazio intramondano dell'esperienza umana, l'infaticabile erranza sarà allora riflesso e ricordo dell'inesauribile serie di occasioni per potersi spingere là dove la Shekhinah attende di essere liberata, ricordando ad ogni passo come Israele tutto sia stato disperso per meglio incontrare le scintille della luce divina sparse in tutte le parti della materia e per risollevarle, con le proprie azioni devote, dalle loro rispettive prigioni.

9 L'eresia sabbatiana, quale «traduzione letterale [...] espressione piena e radicale della cabbala luriana $»^{25}$, prenderà le sue mosse proprio all'interno di una simile tradizione: da un lato, procedendo a una sorta di paradossale prolungamento e conservazione della proposta di Luria, dall'altro muovendosi verso la sua radicalizzazione mediante il collasso del tempo storico entro la narrazione mitologica. In altri termini: restituendo alla vaga carica messianica che il misticismo riconosceva a ciascun ebreo la sua dimensione più urgentemente politica ${ }^{26}$. Se, come ricorda Jesi, «i qabbalisti di Safed demitizzarono radicalmente l'ebraismo nell'istante in cui riuscirono a calarsi in modo totale negli abissi del mito» ${ }^{27}$, ciò fu paradossalmente dovuto proprio alla proiezione delle singole vicende di ogni vita umana su scala cosmica; essi, in altri termini, esaurirono il mito, e in primo luogo il mito relativo alle proprie azioni redentrici, lasciando che ogni cosa trapassasse in esso senza alcuna soluzione di continuità. Intimamente in accordo col tempo del mito, il tempo della storia fu così spogliato della necessità di un qualsiasi intervento divino: rinchiuse nelle pietre e nelle piante, nelle carni degli animali e nei cuori degli uomini, le scintille della luce divina potevano essere riscattate con un compito quotidiano tanto umile quanto impaziente, pressoché indistinguibile dalla condotta di una vita.

10 3. Sarà tale impazienza a giustificare, sull'onda dei più recenti e drammatici avvenimenti storici, l'introduzione nella concezione luriana di quello che Scholem definirà, con evidente riferimento benjaminiano, il suo «carattere distruttivo»: «ciò che ci interessa qui è il modo in cui l'esperienza mistica del contatto dell'uomo con la fonte originaria della vita si condensa sotto forma di un simbolo che contiene in sé la distruzione nichilista dell'autorità ${ }^{28}$. D'altra parte, una volta inaugurato l'aspetto esteriore della redenzione con il manifestarsi del Messia, quale altro modo di 
partecipare al dramma cosmico se non quello di deporre la provvisoria legge dell'esilio in favore della vera legge divina per affrettare, in tal modo, l'avvento della redenzione esterna? Nel momento in cui «molti avvertivano la redenzione come una realtà prima ancora che essa si fosse verificata ${ }^{29}$, nel momento, dunque, in cui l'attesa del compimento messianico subì il richiamo della storia e divenne esperienza del dopodomani, bastò poco affinché quest'ultima potesse essere concepita come prova dell'avvenuto superamento del tempo storico.

11 Non si potrebbe comprendere l'agitazione che ovunque travolse i partecipanti al movimento sabbatiano, senza riferirsi alla «sensazione di miracoloso» ${ }^{30}$ a cui si abbandonarono coloro i quali riconobbero il manifestarsi di una forza del tutto estranea alla situazione dell'esilio, una forza la cui epifania testimoniava inequivocabilmente, ai loro occhi, l'interferenza del tempo del mito con il tempo storico (per i nuovi credenti, «che la Shekhinah (la Presenza divina) si fosse risollevata dalla polvere era ormai un dato di fatto» ${ }^{31}$ ). Ciò «produsse qualcosa che i custodi della tradizione non avevano previsto ma che per lo storico è piuttosto comprensibile: l'esperienza della redenzione come evento storico è anticipata dall'esperienza della redenzione come realtà emotiva ${ }^{32}$. Descrivendo altrove la disposizione peculiare dovuta a una simile esperienza, Scholem non mancherà di ricordare come, «[u]na volta accettata l'idea che la redenzione aveva davvero avuto inizio [...], si presentavano ai credenti situazioni completamente inedite, ed è ben noto quali potenzialità rivoluzionarie si nascondano nell'idea messianica in sé e in ogni tentativo di tradurla in realtà» ${ }^{33}$. L'ultima considerazione è estremamente puntuale, nel momento in cui accenna, secondo i termini di un'opera inaudita di traduzione, alla difficoltà che $\mathrm{i}$ credenti dovettero affrontare nel declinare in maniera radicalmente inedita la propria prassi: se tale epifania confermava le nuove possibilità dischiuse all'uomo, tanto che questi avrebbe dovuto, per mantenersi all'altezza dell'evento, revocare quanto ancora di storico fosse rimasto intatto in seguito all'ingresso del mito nel mondo, come tradurre nella pratica quotidiana e nel tempo storico l'avvenimento eccezionale di cui si era partecipi? Quale nuova prassi liturgica avrebbe dovuto essere compiuta per mantenersi in sintonia con il «miracolo "irragionevole" del tempo di festa [...] la contemplazione dell'epifania miracolosa $»^{34}$ ?

12 Tra i sabbatiani vi furono certamente credenti dalle posizioni più moderate, i quali, al pari di Avrahàm Peretz, reputarono i tempi non ancora maturi per un definitivo abbandono della tradizione, affermando al contrario che la nuova legge sarebbe divenuta interamente percorribile solo dopo che il Messia avesse fatto ritorno dalla sua discesa entro il regno delle qelippot; altri, certi della propria esperienza e radicali nella propria posizione, si convinsero ben presto che la venuta di Tzevì avesse inaugurato un tempo nuovo, in attesa di un suo equivalente nell'ambito della pratica religiosa. Da qui l'istituzione di una «pratica antinomica che è rituale, festa procedente dall'entusiasmo messianico e al tempo stesso testimonianza messianica» ${ }^{35}$, sospensione rivoltosa della temporalità propria al mondo irredento e riconoscimento, senza alcuna riserva, del compimento agognato:

Il tempo del mito era riconosciuto come effettivo tempo storico, già presente o di molto prossimo avvento. Non sarebbe esatto dire che nel tempo storico erano così calate le immagini e le prospettive del mito, innanzitutto del mito messianico. Era invece il tempo del mito che diveniva senza residuo tempo storico: l'utopia era tale, poiché il programma non si fondava su alcun precedente e su alcun rapporto di causalità relativi al tempo storico che non fosse tempo del mito. L'utopia era la 
"nuova legge" che non procedeva dall'antica, ma antinomicamente e paradossalmente cancellava l'antica. Lo spazio aperto e prospetticamente configurato dal paradosso antinomico era spazio di utopia, ma anche spazio storico [...]. A questo punto, si apriva una dimensione prospettica di utopia storicizzata che poteva accogliere, e anzi era per sua natura destinata ad accogliere, una componente eversiva. ${ }^{36}$

13 Nel sottolineare la componente antinomica propria all'eresia, si dovrebbe tuttavia evitare di compiere l'errore di esagerarne l'elemento eversivo, come se il senso ultimo del sabbatianesimo fosse consistito soltanto in questa palese destituzione dei valori mondani e dell'autorità che fino ad allora ne garantiva la validità. Al contrario, per i suoi seguaci non si trattava affatto di negare l'autorità della Legge, bensì di destituire definitivamente una Torah ormai reputata inattuale perché a suo tempo concessa a un mondo irredento, facendo forza sull'attuale vigenza di una Torah all'altezza della compiuta redenzione: rievocando le azioni trasgressive di Tzevì, Jesi non scorderà di specificare come «la legge che egli infrangeva doveva essere infranta affinché si instaurasse la nuova legge del nuovo regno, ma era ancora la legge» ${ }^{37}$. Ha ragione in tal senso Scholem quando, stemperando il presunto carattere anarchico del movimento, specifica che «queste strutture mistiche sono interamente condizionate dai simboli dell'autorità religiosa tradizionale ${ }^{38}$, dai simboli del potere, tanto che l'esperienza mistica rischia ad ogni passo di rovesciare il suo portato rivoluzionario in un atto di conservazione e risolversi così in «una conferma dell'autorità religiosa sotto cui vive; la sua teologia e i suoi simboli vengono proiettati nelle sue esperienze mistiche, ma non ne derivano ${ }^{39}$. Non è forse questo il cuore di quella relazione produttiva che ogni esperienza mistica instaura con la tradizione che contesta e in cui non cessa di rifluire? È come se la funzione di una tale relazione non fosse altro che quella di riposizionare ogni tentativo di trasvalutazione all'interno della tradizione, come suo capitolo ben inserito in un continuum che è riannodato a ogni eventuale interruzione: quale veicolo d'istanze sovversive, esso finisce così per divenire un mero operatore di rinnovamento della medesima tradizione, la quale si dimostra ancora una volta capace di recuperare entro di sé ogni contestazione così come ogni deviazione.

In tal modo, anche il legame tra l'esperienza sabbatiana e quel carattere distruttivo, nichilista o anarchico, che Scholem ha voluto individuare come punto di rottura con la precedente tradizione, appare sotto una luce del tutto differente. Nichilista pare infatti la forma di azione propria ai sabbatiani - i quali ritennero di esperire il Tiqqun e pertanto credettero senza riserve nella necessità di una nuova forma di osservanza solo se giudicata con gli occhi di colui che, non toccato da una simile esperienza, avrà preferito prestare credito al tempo irredento della storia. D'altra parte, volendo affrettare l'evento della restaurazione, i sabbatiani - «autentici credenti, i quali avevano trovato nella promessa di un'utopia anarchica quella redenzione che era stata loro negata dall'ebraismo rabbinico $»^{40}$ - si trovarono letteralmente fuori tempo, vittime di una tensione anticipatoria che portò i loro stessi comportamenti in disaccordo fatale con la storia: anziché anticipare e favorire il compimento visibile della redenzione, essi finirono piuttosto per arroccarsi entro un cerchio chiuso di fedeltà all'evento.

Poiché l'epifania della redenzione si era consumata nello spazio di un istante, la sfida posta ai sabbatiani consistette nel garantire a tale istante una sua durata ininterrotta, aprendo il tempo storico al gesto di una nuova fondazione. La discordanza tra i due tempi fu tale che la certezza della nuova fondazione dovette presto confrontarsi con l'assenza di ogni manifestazione esteriore e politica, corollario del processo cosmico di 
redenzione: nessuno dei credenti, infatti, si sarebbe mai aspettato «che avrebbe potuto generarsi un conflitto tra il simbolo e la realtà che esso veniva a simboleggiare. [...] $\mathrm{Ne}$ derivò che tutte le dottrine sabbatiane furono orientate verso un solo fine comune: costruire un'ideologia che fosse in grado di gettare luce sull'abisso apertosi tra la realtà interiore e quella esteriore, che aveva cessato di essere il suo simbolo e non era più in grado di consentire a coloro che avevano questa nuova sensazione la tensione tra interiorità ed esteriorità $\rangle^{41}$. Ricorrendo a una terminologia straordinariamente prossima a quella impiegata da Jesi per descrivere lo scacco del movimento spartachista, a sua volta Scholem riconduce lo scacco dei sabbatiani all'impossibilità di vivere lo scarto incommensurabile, sul versante del proprio agire, fra il segreto a cui essi erano ammessi a partecipare e la storia ancora aggrappata all'aspetto irredento del mondo: «Poiché fu intrapreso prima del tempo, questo tentativo rimase impigliato nel meccanismo della rivolta e fu soffocato sul nascere $»^{42}$. Cosa accade, allora, quando l'epifania rischia di risolversi in un miraggio, o tutt'al più in un privilegio personale, nel momento in cui la comunità stessa, pronta a celebrare l'ultima festa, si trova infranta proprio là dove parte di essa aveva intravisto la sua stessa salvezza? Cosa accade quando un simbolo crolla sotto il peso della sua inattuabilità, nonché della sua inattualità?

16 4. È stata Hannah Arendt colei che meglio ha saputo cogliere l'ipoteca del mito sull'azione di riscatto intentata dal movimento sabbatiano. In un saggio del 1948, la filosofa propone di rintracciare, sul crinale della torsione operatasi nel sabbatianesimo, la persistenza dell'efficacia del mito nei due momenti di accordo e frattura rispetto al tempo della storia: «Un aspetto degno di nota di questo mito dell'esilio è che serviva a due scopi contrapposti: con la sua interpretazione mistica dell'esilio come azione piuttosto che come patimento, poté spronare il popolo ad affrettare la venuta del Messia e fece sì che si verificasse nel movimento sabbatiano "una stupefacente manifestazione di tutte quelle forze cui doveva l'origine e l'affermazione". Ma dopo il declino di questo movimento, esso provvide ugualmente bene ai bisogni della gente disillusa che, avendo perso la speranza messianica, voleva una nuova e più generica giustificazione dell'esilio, della sua inerte esistenza e della sua mera sopravvivenza» ${ }^{43}$. La mitologia dell'esilio, esasperata dal sabbatianesimo fino a farsi coincidenza con la mitologia relativa all'agire storico, sopravvisse allo scacco in cui quest'ultimo finì per incorrere, entro una forma non più capace di porsi come serbatoio di scintille rivoluzionarie, bensì come cifra segreta della propria condizione storica. Se, come ricorda Elettra Stimilli, «il legame tra fenomeno religioso e nichilismo non solo non si risolve nel processo di secolarizzazione, ma esso, piuttosto, è così profondo da toccare il cuore stesso di ciò che, almeno nella tradizione monoteistica, si intende quando si parla di religione: vale a dire il rapporto tra Dio e mondo» ${ }^{44}$, ben si comprende come lo sfibrarsi di tale rapporto non possa che comportare un'identica proiezione sul piano del legame fra uomo e mondo. Simile in questo a ogni altro gesto di sospensione del tempo storico in accordo con le coordinate del mito, anche la rivolta sabbatiana portò, in ultima istanza, a una nuova forma di sfiducia nei confronti del tempo storico stesso, sostituendo all'iniziale contestazione una radicale disaffezione. La tanto attesa discontinuità tra il nuovo mondo redento e il mondo storico non si produsse nei termini di una deposizione del secondo da parte del primo, ma come una sorta di frattura nell'intima connessione dei due: anziché essere revocato dall'arrivo del messia, il tempo della storia continuava a persistere esteriormente, infrangendo le attese di coloro che avevano operato in favore della sua sospensione. L'irruzione del Messia, 
quale segno della compiuta redenzione, entro un mondo che continuava ostinatamente a presentarsi come irredento, lasciava i credenti alle soglie di un bivio da percorrere sino in fondo: rinunciare al mito e così negare l'esperienza compiuta in sintonia con esso, o riconoscere e giustificare la compresenza tra due temporalità differenti e contrastanti.

È proprio nel solco inaugurato da una simile frattura, e non tanto nel comportamento antinomico di Tzevì, che deve essere rinvenuto il tratto propriamente nichilista del movimento: "[s]i era aperto un abisso tra la realtà interiore e quella esteriore. Da quando i sabbatiani decisero unanimemente che la verità stava dalla parte della realtà interiore, non c'è da stupirsi che venissero concepite teorie volte a svalutare al massimo la realtà esteriore. Su questo punto il messianesimo si mutò in nichilismo» ${ }^{45}$. Anziché aver prodotto un definitivo rinnovamento della situazione politica e sociale degli ebrei, e ancor meno una definitiva redenzione su scala cosmica, l'acuirsi della tensione tra un tempo della storia, pressoché inalterato, e l'esperienza di un tempo della redenzione, ormai compiuto, avrebbe causato un ripiegamento interiore con il quale anche il mito della missione del Messia nella storia, così come la sua stessa figura ${ }^{46}$, finì ben presto per scolorire e dissolversi. Solo situandosi all'interno di una simile frattura tra Dio e mondo, e tra mondo e uomo, è possibile comprendere la scandalosa assenza di qualsiasi elemento positivo in un movimento che, sviluppatosi a partire dall'impazienza di una scarica messianica, non seppe offrire alcun oltrepassamento valorizzante rispetto all'angusta situazione storica in corso: «Il sabbatianesimo come eresia mistica doveva sorgere quando [...] si produsse una frattura tra quelle due scene del dramma della redenzione, quella mistica nell'anima, e quella esterna rappresentata simbolicamente nella storia. L'esperienza interna e l'esterna, l'aspetto interno e l'esterno della redenzione ora improvvisamente e drammaticamente si divisero l'uno dall'altro» ${ }^{47}$. Una volta infranto il segreto accordo tra redenzione cosmica e redenzione storica, con la prima già attuata e la seconda che tardava a giungere, il conflitto tra mondo esterno e mondo interno, tra fallimento del mito nella storia e verità del mito occultata dalla storia, avrebbe presto portato «a svalutare l'azione esteriore, compiuta secondo le norme del vecchio mondo, e a mettere al suo posto un'azione segreta, interna corrispondente alla vera fede» ${ }^{48}$. Sarà la perseveranza nell'azione segreta, fedele al tempo del mito e in quanto tale in evidente contrasto con quello della storia, a provocare l'emergere di qualcosa come una modalità terza di esperire il tempo: un tempo misterico come tempo del segreto accordo con il mito entro la storia, un tempo del segreto, dunque, proprio a coloro che, toccati dalla rivelazione del mito, restano tuttavia esiliati entro il tempo della storia. $\mathrm{Si}$ tratta, in altri termini, dell'inaugurazione di quel tempo appropriato a ciò che Scholem chiama, in maniera alquanto suggestiva ma senza nulla sottrarre alla precisione, il destino apparentemente marranico di chi vive «il crepuscolo intermedio della redenzione ${ }^{49}$. Come se il fatto di vivere un simile crepuscolo liminare, quale unica temporalità concessa entro l'ambito della storia, fosse, come ha ricordato Arendt, già di per sé giustificazione dell'esilio, nonché della sua ineluttabilità.

Più radicale di Scholem, Jesi sostituirà all'immagine del crepuscolo quelle della tenebra e dell'oscurità, «materia buia» che «acquista la gravità di un destino insito nell'organismo umano» ${ }^{50}$. Tornano qui alla mente le riflessioni già condotte in Spartakus, là dove la giustificazione dell'esistente - «l'umano ricorso al mito per lenire il dolore $»^{51}$ - finisce giocoforza per farsi ancella di una immobilità tanto esistenziale quanto destinale, ossia di una immobilità tanto più esistenziale quanto più ascritta al 
già da sempre dato del destino. D'altra parte, una volta sviluppata la «consapevolezza del segreto contrapposto alla storia, del tempo del segreto contrapposto al tempo della storia ${ }^{52}$, non sarà difficile scorgere come, entro l'orizzonte dischiuso da una simile opposizione, la temporalità dell'esilio così giustificato abbia costituito qualcosa come un tempo del riparo dalla storia, una riserva di tempo - nel doppio senso del termine da opporre a quest'ultima. È ancora una volta l'eterno presente del mito a ergersi quale freno nei confronti di ogni possibile conflitto della storia - in essa benché contro di essa. Ed è ancora una volta la presunta epifania del mito, con la sua realtà immobile, a esigere a sua volta l'epifania di una virtù che il credente pagherà a caro prezzo, ossia con quel «deliberato straniamento dal tempo storico» ${ }^{53}$, con quella ostilità radicale tanto al mondo quanto alla storia, che ha da sempre accompagnato la consolazione della vittima e dell'esiliato ogni qual volta essi hanno ammantato la propria condizione con una qualche sorta di trasvalutazione metastorica.

\section{BIBLIOGRAFIA}

ARENDT 1998: H. Arendt, «Una rilettura della storia ebraica» (ed. or. 1948), in ead., Ebraismo e modernità, trad. it., Milano 1998, 145-156.

BENJAMIN 2006: W. Benjamin, «[Appendice a La Parigi del Secondo Impero in Baudelaire]», in Id., Opere complete. VII. Scritti 1938-1940 (ed. or. Frankfurt am Main 1972-1989) , tr. it., Torino 2006.

BIALE 2019: D. Biale, Il maestro della cabala. Vita di Gershom Scholem (ed. or. New Haven-London 2017), Roma 2019.

CAVALLETTI 2013: A. Cavalletti, «“Quasi uno houyhnhnm”. Furio Jesi e l'ebraismo», Scienza e politica 48 (2013), 95-101.

GUERRA 2009: G. Guerra, «L'eresia mistica. Il messianismo di Sabbetay Sevi», in I. Bahbout, D.

Gentili, T. Tagliacozzo (a cura di), Il messianismo ebraico, Firenze 2009, 59-70.

IDEL 2004: M. Idel, Mistici messianici (ed. or. New Haven-London 1998), tr. it., Milano 2004.

JACOBSON 2001: E. Jacobson, «Anarchismo e tradizione ebraica: Gershom Scholem», in A. Bertoli (a cura di), L'anarchico e l'ebreo. Storia di un incontro, Milano 2001, 55-75.

JACOBSON 2003: E. Jacobson, Metaphisics of the Profane. The Political Theology of Walter Benjamin and Gershom Scholem, New York 2003.

JESI 1972: F. Jesi, Mitologie intorno all'illuminismo, Milano 1972.

Jesi 1980: F. Jesi, «L'esploratore della Kabbalah. Scholem e i mistici», La Stampa, 7 marzo 1980, 3.

JESI 1991: F. Jesi, Mitologie intorno all'illuminismo (ed. or. Milano 1972), Bergamo 1991.

JESI 2000: F. Jesi, Spartakus. Simbologia della rivolta (1969), Torino 2000.

JESI 2002: F. Jesi, Esoterismo e linguaggio mitologico. Studi su Rainer Maria Rilke (ed. or. Firenze 1976), Macerata 2002. 
JESI 2010: F. Jesi, «Sul fatto miracoloso» (ed. or. Torino 1959), in Id., «La ceramica egizia» e altri scritti sull'Egitto e la Grecia (1956-1973), Torino 2010, 245-252.

JESI 2011: F. Jesi, Cultura di destra (ed. or. Milano 1979), Roma 2011.

JESI 2013: Lettera di Furio Jesi a Gershom Scholem del 26 novembre 1966, Scienza e politica 48

(2013), 107.

LUCCA 2013: E. Lucca, «Ateismo e profondità dell'essere. Un breve scambio epistolare tra Furio Jesi e Gershom Scholem», Scienza e politica 48 (2013), 111-116.

MANERA 2012: E. Manera, Furio Jesi. Mito, violenza, memoria, Roma 2012.

MOSÈs 2006: S. Mosès, L'Ange de l'Histoire, Paris 2006.

SCHOLEM 1980: G. Scholem, La Kabbalah e il suo simbolismo (ed. or. Zürich 1960), trad. it., Torino 1980.

SCHOLEM 1993: G. Scholem, Le grandi correnti della mistica ebraica (ed. or. Jerusalem 1941), trad. it., Torino 1993.

SCHOLEM 1998: G. Scholem, «La trasgressione come adempimento della miswah» (ed. or. Jerusalem 1936-1937), in Id., Mistica, utopia e modernità. Saggi sull'ebraismo, trad. it., Genova 1998, 49-146.

SCHOLEM 2001: G. Scholem, Sabbetay Sevi. Il messia mistico (ed. or. Frankfurt am Main 1957), trad. it., Torino 2001.

SCHOLEM 2008: G. Scholem, L'idea messianica nell'ebraismo e altri saggi sulla spiritualità ebraica (ed. or. Frankfurt am Main 1971), trad. it., Milano 2008.

SCHOLEM 2014: G. Scholem, «La metamorfosi del messianismo eretico sabbatiano in nichilismo religioso nel XVIII secolo» (ed. or. Frankfurt am Main 1963), in id., Le tre vite di Moses Dobrushka, trad. it., Milano 2014, 139-158.

SCHOLEM 2016: G. Scholem, Il nichilismo come fenomeno religioso (ed. or. Frankfurt am Main 1984), trad. it., Firenze 2016.

STIMILLI 2000: E. Stimilli, «Il nichilismo sabbatiano come fenomeno politico», in R. Esposito, C. Galli, V. Vitiello (a cura di), Nichilismo e politica, Roma-Bari 2000, 247-267.

\section{NOTE}

1. BENJAMIN 2006, 213.

2. JESI $1991,17-41$.

3. Cfr. JESI 2000.

4. JESI 1991, 32.

5. Ibid.

6. MANERA 2012, 66.

7. JESI 1991, 45 (corsivo dell'autore).

8. Una consonanza che, d'altra parte, trova riscontro anche nei diversi tentativi compiuti da Jesi per instaurare un dialogo con Scholem, a partire dal breve scambio di lettere inaugurato nel 1966 su iniziativa dello stesso Jesi (JESI 2013) fino alla recensione, datata 1980, del volume La Kabbalahe il suo simbolismo (JESI 1980). Benché ad oggi non sia disponibile alcuna ricostruzione esaustiva del rapporto tra i due studiosi (così come del rapporto tra Jesi e, più in generale, la tradizione 
ebraica), alcuni passi sono stati compiuti da Andrea Cavalletti ed Enrico Lucca. Cfr. CAVALLETTI 2013; LUCCA 2013.

9. JESI 1972, 9.

10. Sull'ipotesi di considerare la tradizione cabalistica come una forma di ripresa mitologica entro una religione monoteistica, si rimanda alle ricostruzioni proposte da MOSÈs 2006, 267-368, e BIALE 2019, 123-144.

11. Sull'intimo nesso che lega, nel pensiero di Scholem, teologia politica, anarchismo e messianismo, si vedano JACOBSON 2001, JACOBSON 2003, 52-84.

12. SCHOLEM 1993, 308.

13. Ibid.

14. Sul sabbatianesimo come movimento, si rimanda a IDEL 2004, 240 .

15. SCHOLEM 1980, 37.

16. SCHOLEM 1993, 301.

17. GUERRA 2009, 62 .

18. Cfr. SCHOLEM 2001, 785-787.

19. Ivi, 35.

20. SCHOLEM 1998, 63.

21. SCHOLEM 1993, 271.

22. Cfr. SCHOLEM 1980, 120: «che cosa costituiva, nell'ebraismo rabbinico, il carattere non mitico della legge? La risposta è chiara: il distacco della legge da ogni esecuzione cosmica».

23. SCHOLEM 1993, 271.

24. Ivi, 287. Sulla teodrammatica di Luria, quale «reazione mitica all'interno di regioni che il pensiero monoteistico aveva strappato al mito con infinita fatica», si veda in particolare SCHOLEM 1980,125 ss.

25. STIMILLI 2000, 250.

26. Sul movimento sabbatiano come «ultima importante esperienza politica ebraica», si rimanda alle considerazioni di ARENDT 1998, 145 ss.

27. JESI 1991, 31.

28. SCHOLEM 1980, 37-38.

29. SCHOLEM 1993, 300.

30. JESI 2010, 249.

31. SCHOLEM 1998, 64.

32. SCHOLEM 2008, 70 .

33. SCHOLEM 2014, 142.

34. JESI 1991, 30.

35. Ivi, 46. La posizione di Jesi qui espressa sembrerebbe costituire un'esplicita variazione di quanto già scritto da Scholem, là dove descrive il compimento della trasgressione come «un rituale, una festa del singolo o di un intero gruppo, qualcosa di insolito che ha origine da uno stato di esaltazione e che ne è, al tempo stesso, la testimonianza» (sCHolem 1993, 399).

36. JESI 1991, 71-72.

37. JESI 2011, 74.

38. SCHOLEM 1980, 12

39. Ibid. E questo benché, in un altro saggio, Scholem abbia descritto il nichilismo religioso come quel fenomeno che «riconosce la sfera religiosa, ma nega radicalmente l'autorità che a suo avviso si arroga il diritto di controllare tale sfera. Esso non punta al consolidamento di strutture nuove al posto di quelle antiche, ma alla loro distruzione» (sCHOLEM 2016, 15). D'altra parte, sarà lo stesso autore a insiste altrove sulla difficoltà di pensare, e ancor più di promuovere, qualcosa come una «totale negazione» di ciò a cui il termine "tradizione" allude: «che cosa resta anche dopo la 
rottura? La rottura di una tradizione è davvero una rottura? Non sarà che la tradizione riesce in qualche modo a sussistere in formule e configurazioni nuove sebbene in apparenza la metamorfosi venga rifiutata?» (SCHOLEM 2008, 59).

40. SCHOLEM 2016, 74.

41. SCHOLEM 1998, 64-65.

42. SCHOLEM 2014, 158.

43. ARENDT 1998, 153.

44. STIMILli 2000, 247. Non manca, tuttavia, chi ha saputo scorgere nel sabbatianesimo un'importante tappa nel processo di secolarizzazione delle coscienze europee culminante con l'«espulsione del "religioso"» in tempo di illuminismo (GUERRA 2009, 69). Al contrario, nell'economia del presente lavoro, resta centrale la qualità eminentemente religiosa di quell'esperienza del tempo storico che per Jesi ha costituito la cifra stessa del movimento sabbatiano. D'altra parte, la più evidente conseguenza del rovesciamento del nichilismo sabbatiano in illuminismo andrebbe piuttosto ricercata in quella sua capacità di convogliare le energie utopiche scatenate dal messianismo verso quella nuova forma di religiosità propriamente politica che troverà libero corso, di lì a breve, nel solco inaugurato dalla Rivoluzione francese. Esemplare in tal senso è la figura di Moses Dobrushka, seguace del frankismo nonché nipote acquisito dello stesso Frank, convertitosi al cattolicesimo, per poi abbracciare la causa giacobina e finire sul patibolo, nel 1794, a fianco di Danton (cfr. SCHOLEM 2014).

45. SCHOLEM 1998, 96.

46. Cfr. ivi, 117: «La stessa figura di Shabbetay Sevi, col tempo, cambiò i suoi contorni e divenne un mito: la prospettiva storica perse rilevanza sino a svanire e rimase solo la figura dell'eroe mitico, che aveva trovato una via nuova e aveva dato inizio a un'era nuova».

47. SCHOLEM 1993, 314.

48. Ivi, 324 .

49. Ivi, 319.

50. JESI 2000, 97-99.

51. Ivi, 90 .

52. JESI 2002, 117.

53. JESI 1972, 9.

\section{RIASSUNTI}

A partire dall'esame degli scritti dedicati da Furio Jesi al movimento sabbatiano, questo articolo si propone di indagare le osservazioni jesiane attorno alla rivolta nella sua qualità di fenomeno religioso. Aprendosi a un dialogo con differenti letture della medesima eresia, quali quelle avanzate da Gershom Scholem, Jesi cerca di superare i limiti imposti da un approccio puramente storico, arricchendo le proprie osservazioni di implicazioni politiche e filosofiche, nonché rendendo la propria analisi un preteso per una più ampia riflessione sull'intima connessione tra politico e religioso, tra tempo della storia e tempo del mito.

Taking into account the writings of Furio Jesi consecrated to the Sabbatian movement, this study aims to investigate the observations made by Jesi about the revolt as a religious phenomenon. Engaging in a dialogue with different analysis of the same heresy, like those leaded by Gershom 
Scholem, Jesi tries to overpass the limits imposed by a strict historical approach, filling his reading with philosophical and political implication and making his work a pretext for a wider reflection about the intimate connection between religious and political, history and myth.

INDICE

Keywords : Jesi Furio, revolt, messianism, tradition, Scholem Gershom, sabbatianism

Parole chiave : Jesi Furio, rivolta, messianismo, tradizione, Scholem Gershom, sabbatianesimo

\section{AUTORE}

\section{MARCO TABACCHIN}

Università di Verona

Dipartimento di Scienze Umane

Lungadige Porta Vittoria 17

37129 Verona

marco.tabacchini(at)univr.it 